\title{
Summarizing health-related quality of life (HRQOL): development and testing of a one-factor model
}

\author{
Shaoman Yin ${ }^{1 *}$ DD, Rashid Njai ${ }^{2}$, Lawrence Barker ${ }^{2}$, Paul Z. Siegel ${ }^{2}$ and Youlian Liao ${ }^{2}$
}

\begin{abstract}
Background: Health-related quality of life (HRQOL) is a multi-dimensional concept commonly used to examine the impact of health status on quality of life. HRQOL is often measured by four core questions that asked about general health status and number of unhealthy days in the Behavioral Risk Factor Surveillance System (BRFSS). Use of these measures individually, however, may not provide a cohesive picture of overall HRQOL. To address this concern, this study developed and tested a method for combining these four measures into a summary score.

Methods: Exploratory and confirmatory factor analyses were performed using BRFSS 2013 data to determine potential numerical relationships among the four $\mathrm{HRQOL}$ items. We also examined the stability of our proposed one-factor model over time by using BRFSS 2001-2010 and BRFSS 2011-2013 data sets.

Results: Both exploratory factor analysis and goodness of fit tests supported the notion that one summary factor could capture overall HRQOL. Confirmatory factor analysis indicated acceptable goodness of fit of this model. The predicted factor score showed good validity with all of the four HRQOL items. In addition, use of the one-factor model showed stability, with no changes being detected from 2001 to 2013.

Conclusion: Instead of using four individual items to measure HRQOL, it is feasible to study overall HRQOL via factor analysis with one underlying construct. The resulting summary score of HRQOL may be used for health evaluation, subgroup comparison, trend monitoring, and risk factor identification.
\end{abstract}

Keywords: Health-related quality of life, Summary score, Factor analysis

\section{Background}

Health-related quality of life (HRQOL) is a useful indicator of overall health because it captures information on the physical and mental health status of individuals, and on the impact of health status on quality of life [1, 2]. HRQOL is usually assessed via multiple indicators of self-perceived health status and physical and emotional functioning. Together, these measures provide a comprehensive assessment of the burden of preventable diseases, injuries, and disabilities [3].

To assess and measure HRQOL at the state and national levels, the Centers for Disease Control and Prevention (CDC) developed a set of four "core" questions (CDC HRQOL-4): (1) Would you say that in general your health

\footnotetext{
* Correspondence: wso3@cdc.gov

'SciMetrika, LLC, 100 Capitola Drive, Durham, NC 27701, USA

Full list of author information is available at the end of the article
}

is excellent, very good, good, fair, or poor? (2) Now thinking about your physical health, which includes physical illness and injury, for how many days during the past 30 days was your physical health not good? (3) Now thinking about your mental health, which includes stress, depression, and problems with emotions, for how many days during the past 30 days was your mental health not good? (4) During the past 30 days, for about how many days did poor physical or mental health keep you from doing your usual activities, such as self-care, work, or recreation? [3-5].

These four items, which have demonstrated good retest reliability, validity, and responsiveness [6-8], have been included in the Behavioral Risk Factor Surveillance System (BRFSS) in all 50 states since 1993. In addition, the four items have also been included in other national surveys (e.g., National Health and Nutrition Examination 
Survey (NHANES), Medicare Health Outcome Survey) and in various chronic disease assessments $[7,9,10]$. CDC HRQOL-4 account for similar variance as the PatientReported Outcome Measurement Information System (PROMIS) items (e.g., SF-36) [11-13]. However, the CDC items appear more appropriate for assessing burden of disease for chronic conditions and are brief and easily interpretable [11].

In 1995, CDC added five additional questions related to quality of life to BRFSS, as part of an optional module. The new questions asked about days experiencing pain, feeling sad or depressed, feeling worried or anxious, not getting enough rest, or feeling healthy. However, the optional module was only used in a limited number of states and years.

To assess HRQOL comprehensively, public health professionals have sought a means to summarize these HRQOL measures. To combine the information on physically and mentally unhealthy days, some researchers have summed the two measures in CDC HRQOL-4 to create an Unhealthy Days Index, with the sum of the two items being truncated at 30 days $[3,14,15]$. This approach assumes an independent relationship between the two kinds of days.

Another approach is to view HRQOL as a latent (hidden) construct that can be quantified through factor analysis. Factor analysis is a method for detecting relationships among variables, which often reduces the number of variables. Previous studies found strong associations among the CDC HRQOL-4 questions, suggesting that these items may be suitable for factor analysis [4]. Toet and colleagues found good internal consistency of the four measures (the Cronbach's alpha for the three unhealthy day measures was 0.77 ; a Cronbach's alpha of 0.70 or more is usually considered acceptable [16]) [13]. Horner-Johnson and colleagues, on the other hand, found a relatively poor consistency between the mentally unhealthy day item and the three other items based on "the Cronbach's alpha increase if item removed" test [17]. They compared two alpha values: one based on all items; the other based on remaining items after a test item was removed. This analysis relies on the premise that if the test item value increases, this may indicate poor consistency of the removed item. However, due to the lack of a clear cutoff value for the increase, it is a somewhat subjective choice to remove a single item measure, especially for situations in which the increase in the alpha values is minimal. Horner-Johnson and colleagues found only a very slight increase (e.g., 0.001 when using BRFSS 2002 data), which may not be enough to undermine the internal consistency of the mentally unhealthy day item with other HRQOL items [17]. Raykov and colleagues warned that the Cronbach's alpha if item is removed test can be misleading for selecting construct components $[18,19]$.

Two studies have conducted HRQOL factor analysis using the CDC HRQOL-4 plus the five optional HRQOL module questions [7, 17]. Using data from BRFSS (2001 and 2002), both studies demonstrated that the nine HRQOL questions have good internal consistency and could be reduced to two latent factors that correspond to the physical and mental health aspects of HRQOL. However, data from the optional BRFSS module were only available for a few states and years, which limits the application of these models in tracking HRQOL over the years or assessing HRQOL at the national level.

This study proposes a method for creating a summary score of overall HRQOL based solely on CDC HRQOL-4. Public health professionals could treat such a consolidated score as a "new" variable that could be used to describe both community and population health, assess health disparities, monitor trends, and identify risk factors of overall HRQOL at the local and/or national levels. Using the 2013 BRFSS data set, the study assesses whether there is an underlying latent construct of HRQOL for the general population, and investigates the possibility of reducing CDC HRQOL-4 to one summary score. It also provides an example of how this type of summary score could be used in trend analysis using BRFSS 2001-2010 and 20112013 data sets.

\section{Methods}

\section{Data sources}

The BRFSS is a state-based random-digit-dialed telephone health survey system. The survey annually collects data from non-institutionalized civilian adults $(\geq 18$ years of age) about their health-related risk behaviors, chronic health conditions, and use of preventive services [20]. Starting in 2011, BRFSS changed its weighting methodology and added cellular telephone users to its samples. Due to these changes, caution should be used when comparing BRFSS data from before and after 2011 [21]. In our analyses, we included two groups of data sources: BRFSS 2013 data (as an experimental study for factor analysis) and BRFSS 2001-2013 data sets (to assess model stability and perform trend analysis, one for 2001-2010 data sets and another for 2011-2013 data sets). Data on the four HRQOL questions were available from all states for every year, except 2002, when data were available from 22 states only.

\section{Statistical analysis}

To study the underlying structure of the CDC HRQOL-4, we conducted Cronbach's alpha test, exploratory factor analysis (EFA), and confirmatory factor analysis (CFA) using BRFSS 2013 data. We then assessed the stability of the resulting model over years, and demonstrated its applications for trend analysis using BRFSS 2001-2010 and 2011-2013 data sets.

To analyze the internal consistency or reliability of the CDC HRQOL-4, we performed Cronbach's alpha test (a larger alpha value indicates greater internal 
correlation). We used the traditional cutoff value of 0.70 or higher as being acceptable [16]. To reveal construct dimensions, EFA was used, with factors with an eigenvalue (a number showing how much variance there is for that underlying factor) larger than or equal to 1.0 being considered acceptable [22]. The principal axis factoring with rotation of orthogonal varimax rotation was used, which can accommodate non-normal data distribution [23].

Based on the results of Cronbach's alpha test and EFA, we hypothesized that it would be possible to summarize the CDC HRQOL-4 items by using a single factor. To determine if the model adequately fit the data, we conducted a goodness of fit test using CFA. We used an asymptotically distribution-free method to account for non-normality of the data and ordinal data [24]. Five model fit statistics were used to evaluate model fit: root mean squared error of approximation (RMSEA), comparative fit index (CFI), Tucker-Lewis index (TLI), standardized root mean squared residual (SRMR), and coefficient of determination (CD). We followed commonly accepted criteria regarding goodness of fit: RMSEA $(\leq 0.06)$, CFI and/or TLI ( $\geq 0.95)$, SRMR $(\leq 0.08)$, and CD close to 1 [25]. Using one-factor model regression, we generated HRQOL factor score values. To confirm the validity of the HRQOL factor scores, we compared the mean changes in the HRQOL factor scores with each level of the HRQOL measures.

After establishing the one-factor HRQOL model using BRFSS 2013 data, we assessed model stability over the years using two data sets: BRFSS 2001-2010 (10 years) and 2011-2013 (3 years). To do so, we conducted a series of hierarchical tests including factorial configural invariance (similar factor structure across groups), metric invariance (equivalent factor loadings across groups), and scalar invariance (equivalent intercepts across groups) [26]. In sequencing of these tests (increasing constraints on model parameters), we followed the recommended criteria, which suggest that the more restrictive nested model with a decrease of CFI less or equal to 0.01 be accepted [27, 28]. Next, HRQOL factor scores for the 13 years were generated by model predication. Survey sampling design and weighting were considered in the analyses. The year 2000 US standardized population was used for age standardization. All analyses were conducted using STATA 13.0 statistical software (College Station, TX: StataCorp LP).

\section{Results}

\section{Factor structure}

Using BRFSS 2013 data, we first analyzed the correlation matrix and internal consistency of the CDC HRQOL-4 questions (Table 1). The Cronbach's alpha value of the CDC HRQOL-4 was 0.76, which was within the acceptable range [16]. The alpha change if the item were removed test indicated good consistency within items. Removing the mentally unhealthy day items increased alpha by $1.3 \%$, which is consistent with HornerJohnson's results [6]. EFA (Table 2) showed that a single factor, with an eigenvalue larger than one, explained $99.9 \%$ of the total variance. Therefore, we propose a one-factor HRQOL model for the CDC HRQOL-4.

\section{Factor model}

An initial model with four paths from one factor to the four CDC HRQOL-4 items was first evaluated by CFA. The four items had factor loadings that ranged from 0.46 to 0.87 , larger than the minimal acceptable cutoff value of \pm 0.3 [26]. The goodness of fit statistics indicate that the model is acceptable but could be improved upon $(\mathrm{RMSEA}=0.086, \mathrm{CFI}=0.90, \mathrm{TLI}=0.70, \mathrm{SRMR}=$ $0.03, \mathrm{CD}=0.85$ ). To determine whether the model could be improved, a post-hoc model modification was performed. We found that adding an error correlation path between the physically unhealthy day item and the mentally unhealthy day item substantially improved the goodness of fit between model and data. Thus, a final model was proposed (Fig. 1). The minimal factor loading was increased from 0.46 to 0.54 . The goodness of fit statistics were also greatly improved (RMSEA $=0.039$, $\mathrm{CFI}=0.99, \mathrm{TLI}=0.94, \mathrm{SRMR}=0.01, \mathrm{CD}=0.89)$.

\section{Factor scores}

To quantify the overall HRQOL, weighted factor score values were predicted by the final CFA model. Factor score could be considered as weighted sum scores (multiplying the score of each item into its factor loading and then summing all of them). Figure 2 shows the distribution of predicted factor scores using BRFSS 2013 data, with a larger value indicating better quality of HRQOL. The "skewed left" distribution suggests that the majority of the population is healthy in terms of HRQOL. To check the consistency of HRQOL factor scores with their original measures, we summarized HRQOL factor scores for each level of CDC HRQOL-4 (Table 3). Either in one year or across years, the overall means of HRQOL factor scores decrease as the CDC HRQOL-4 ratings become worse for both male and female adults (we did an analysis stratified by sex, discussed later), indicating the validity of factor scores in representing HRQOL.

\section{Model stability}

To test whether our HRQOL model was stable over time, we examined BRFSS data from 2001 to 2013. Table 4 summarizes the goodness of fit statistics of the model using a series of BRFSS data sets. For all the data sets, whether the combined (2001-2010 or 2011-2013) or individual years were examined, our HRQOL model exhibited acceptable goodness of fit (RMSEA $=0.035-0.05$, CFI $=0.984-0.99$, 
Table 1 Correlation matrix and internal consistency of the CDC HRQOL-4 items, BRFSS 2013

\begin{tabular}{|c|c|c|c|c|c|c|}
\hline & $\begin{array}{l}\text { General health } \\
\text { status }\end{array}$ & $\begin{array}{l}\text { Physically } \\
\text { unhealthy days }\end{array}$ & $\begin{array}{l}\text { Mentally } \\
\text { unhealthy days }\end{array}$ & $\begin{array}{l}\text { Activity } \\
\text { limitation days }\end{array}$ & $\begin{array}{l}\text { Cronbach's alpha } \\
\text { if item removed }\end{array}$ & Change (\%) \\
\hline General health status $^{a}$ & 1 & & & & 0.733 & -3.9 \\
\hline Physically unhealthy days ${ }^{b}$ & 0.52 & 1 & & & 0.651 & -14.6 \\
\hline Mentally unhealthy days ${ }^{c}$ & 0.29 & 0.35 & 1 & & 0.773 & +1.3 \\
\hline Activity limitation days ${ }^{d}$ & 0.43 & 0.65 & 0.44 & 1 & 0.656 & -14 \\
\hline Overall construct & & & & & 0.763 & \\
\hline
\end{tabular}

Would you say that in general your health is excellent, very good, good, fair, or poor?

${ }^{b}$ Now thinking about your physical health, which includes physical illness and injury, for how many days during the past 30 days was your physical health not good?

'Now thinking about your mental health, which includes stress, depression, and problems with emotions, for how many days during the past 30 days was your mental health not good?

${ }^{\mathrm{d} D u r i n g}$ the past 30 days, for about how many days did poor physical or mental health keep you from doing your usual activities, such as self-care, work, or recreation?

$\mathrm{TLI}=0.915-0.938, \quad \mathrm{SRMR}=0.01-0.014$, and $\mathrm{CD}=0.868$ 0.885). To further examine this, we analyzed results from a sequence of hierarchical tests (Table 5). For both of the combined data sets (2001-2010 and 2011-2013), all models had acceptable goodness of fit statistics (RMSEA = 0.02-0.044, CFI $=0.977-0.987$, TLI $=0.925-0.984$, SRMR $=$ 0.011-0.014, and CD $=0.879-0.884)$. The decrease in CFI was no larger than 0.01 for each model pairwise comparison, whether it involved full metric invariance versus full configural invariance, or full scalar invariance versus full metric invariance. These results indicate that the new, single measure of HRQOL has strong measurement invariance, holding full equivalent factor patterns, full equivalent factor loadings, and full equivalence intercepts over the years, from 2001 to 2010, and from 2011 to 2013.

We also further assessed model stabilities across sex and age subgroups (Table 5). Results suggest that the one-factor model has strong measurement invariance across sex, holding full equivalent factor patterns, full equivalent factor loadings, and full equivalence intercepts between male and female adults. When applied to young (18-64) and old (65+) age subgroups, the onefactor model has full configural invariance but the full equivalent factor loadings is not supported as the CFI decrease is larger than 0.01 . However, after releasing the equivalent factor loading constraints for the mentally unhealthy day item, partial metric invariance is tenable.

\section{Model application: trend monitoring}

The one-factor HRQOL model exhibits strong measurement invariance across year subgroups, which allows us to analyze how the mean of HRQOL factor scores changes

Table 2 Exploratory factor analysis of the CDC HRQOL-4, BRFSS 2013

\begin{tabular}{lccc}
\hline Factor & Eigenvalue & $\begin{array}{c}\text { Percentage of } \\
\text { explained variance }\end{array}$ & $\begin{array}{c}\text { Accumulated Percentage } \\
\text { of explained variance }\end{array}$ \\
\hline factor 1 & 1.76 & 99.9 & 99.9 \\
factor 2 & $<0.01$ & 0.1 & 100.0 \\
\hline
\end{tabular}

over years. Figure 3 shows the age-standardized weighted means of HRQOL factor scores predicted for the 20012010 and 2011-2013 periods, respectively. The overall HRQOL scores gradually declined from 2001 to 2004 and, in general, remained stable thereafter through 2010 ( $p<0.001$ for 2001 vs. 2004, adjusted Wald test). Compared with 2011 and 2012, the overall HRQOL scores increased in 2013 ( $p<0.001$ for 2011 vs. 2013, adjusted Wald test). These findings were also confirmed with the changes from the original CDC HRQOL-4 questions (Additional file 1 shows results of CDC HRQOL-4 changes for 2001 vs. 2004, and 2011 vs. 2013).

\section{Discussion}

In this study, we developed and tested a one-factor HRQOL model using a series of BRFSS data sets. To our knowledge, this is the first report of an HRQOL factor analysis based solely on CDC HRQOL-4. Two previous studies, which used data obtained from the optional BRFSS module, proposed a two-factor model [7, 17]. One report used summed z-scores from all items to represent physical and mental health, respectively. However, it did not consider item factor loadings and removed one item due to the cross loading issues [17]. As the CDC HRQOL-4 questions are more commonly used in BRFSS and other surveys, we performed HRQOL factor analysis using only these four items. EFA revealed that the four items could be explained by one underlying factor-a general health factor that encompasses both physical and mental health. As a result, this model could be used to generate a one-factor score that represents the underlying construct of HRQOL.

In addition to EFA, we performed CFA to evaluate our one-factor model with more statistical options such as goodness of fit, modification indices, and measurement invariance tests. Our post-hoc analysis found a negative error correlation path between the physically unhealthy day item and the mentally unhealthy day item. This result may have not only statistical support but also theoretical 


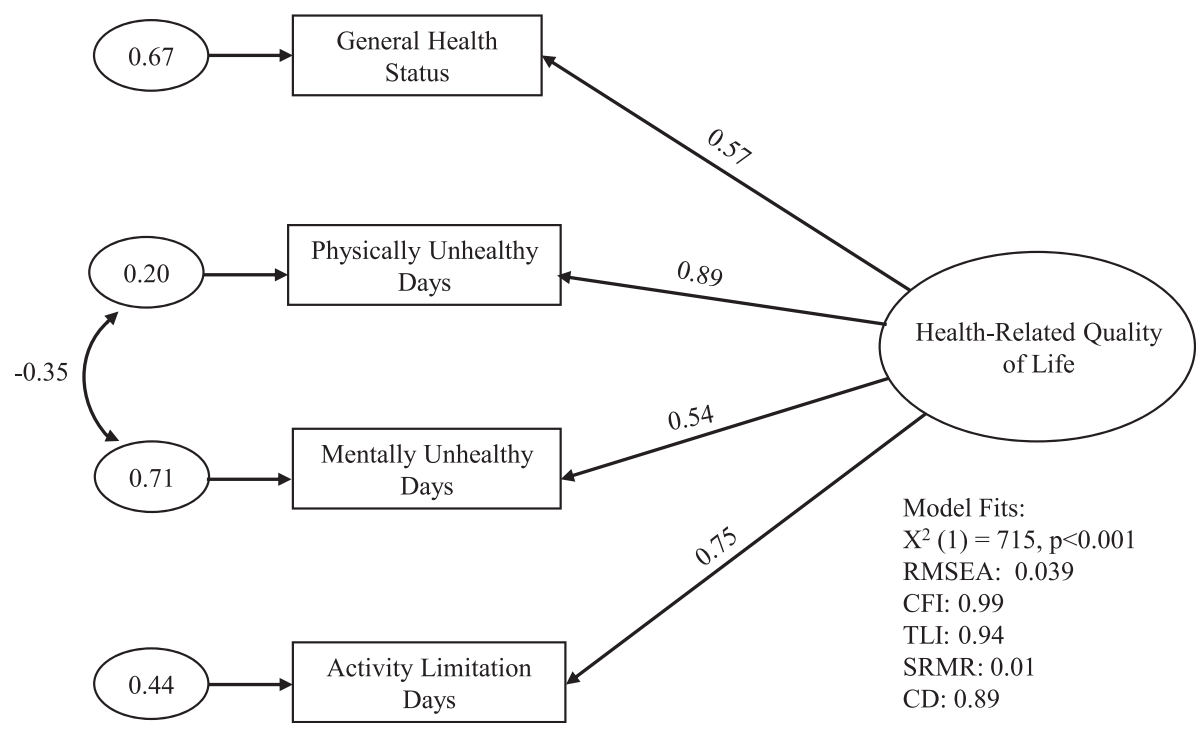

Fig. 1 Final one-factor model for the CDC HRQOL-4, BRFSS 2013. Standardized factor loadings from the latent construct (represented by the large oval) to its measures (represented by rectangles) are shown beside the single-headed arrows. The small ovals represent error variances unexplained by the model. The curved double-headed arrow represents correlations between error variances

meaning. First, research has found that using similar question formats can affect survey responses [29]. The format of the two questions is very similar, which may contribute to the covariance between the two items. Second, our preliminary analysis (not shown) found that some individuals report no physically unhealthy days, but 30 mentally unhealthy days. Our one-factor model may account for this distinction by indicating a negative relationship between the error terms in the measures of physically unhealthy days and mentally unhealthy days.

Our one-factor model showed strong measurement invariance across year and sex subgroups. However, for young and old age subgroups, only partial metric invariance was observed due to different factor loadings on the mentally unhealthy day item. This may suggest that young and old people have different dimensions on mental health aspect, which is in accordance with previous reports [30, 31]. Further studies are needed to show how stable the factor structure is with other demographics, socioeconomic characteristics, and chronic conditions.

Using BRFSS 2001-2010 and 2011-2013 data sets, we demonstrated that our one-factor HRQOL model is stable over time, and could be used to monitor trends in HRQOL with a single summary score. This approach

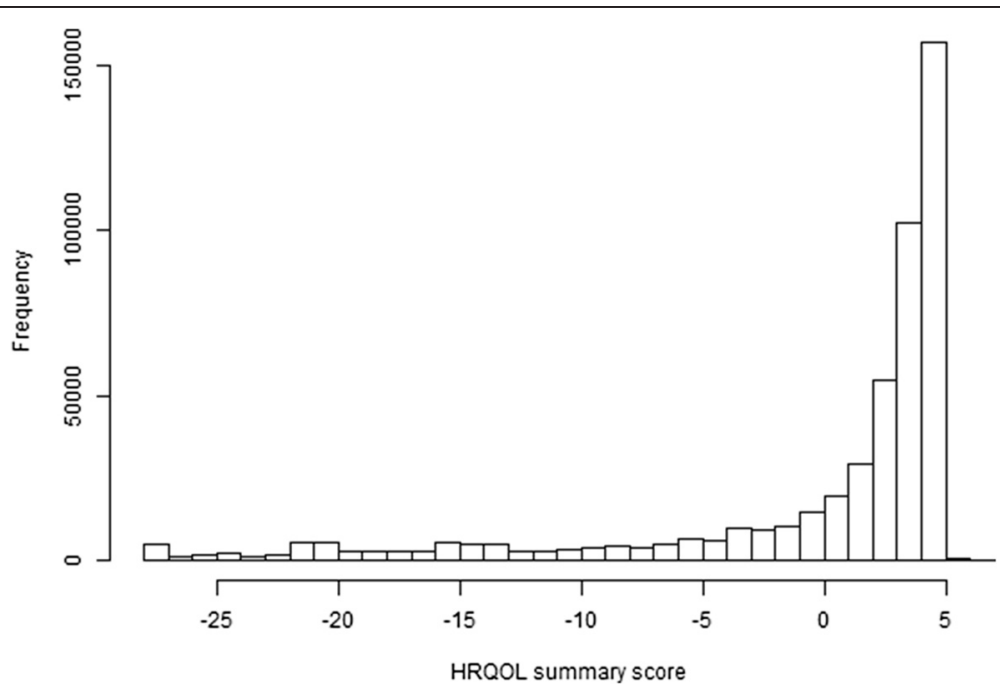

Fig. 2 HRQOL summary score, BRFSS 2013. Histogram shows the distribution of the HRQOL summary score using BRFSS 2013 data set. Larger value means better quality of HRQOL. The "skewed left" distribution suggests that the majority of the population is healthy in terms of HRQOL 
Table 3 The mean values of HRQOL summary scores by the CDC HRQOL-4 measures

\begin{tabular}{|c|c|c|c|c|c|c|c|c|}
\hline \multirow{2}{*}{$\begin{array}{l}\text { BRFSS Data Sets } \\
\text { Sex }\end{array}$} & \multicolumn{2}{|l|}{2005} & \multicolumn{2}{|c|}{$2001-2010$} & \multicolumn{2}{|l|}{2013} & \multicolumn{2}{|c|}{$2011-2013$} \\
\hline & Male & Female & Male & Female & Male & Female & Male & Female \\
\hline \multicolumn{9}{|c|}{ General Health Status } \\
\hline Excellent & 3.10 & 4.04 & 3.14 & 4.05 & 3.32 & 4.17 & 3.35 & 4.18 \\
\hline Very good & 1.81 & 2.58 & 1.84 & 2.59 & 2.09 & 2.84 & 2.13 & 2.84 \\
\hline Good & -0.20 & 0.42 & -0.18 & 0.44 & -0.03 & 0.63 & 0.01 & 0.63 \\
\hline Fair & -6.21 & -5.60 & -6.20 & -5.58 & -6.55 & -5.84 & -6.50 & -5.84 \\
\hline Poor & -16.91 & -16.14 & -16.89 & -16.12 & -17.34 & -16.46 & -17.27 & -16.45 \\
\hline \multicolumn{9}{|c|}{ Physically Unhealthy Days } \\
\hline 0 day & 2.80 & 3.50 & 2.82 & 3.52 & 2.99 & 3.67 & 3.02 & 3.67 \\
\hline 1-10 days & -0.04 & 0.71 & -0.01 & 0.73 & 0.07 & 0.79 & 0.11 & 0.79 \\
\hline 11-20 days & -7.98 & -7.10 & -7.96 & -7.08 & -7.85 & -6.98 & -7.79 & -6.97 \\
\hline 21-29 days & -15.81 & -14.72 & -15.79 & -14.71 & -15.64 & -14.60 & -15.57 & -14.57 \\
\hline 30 days & -21.17 & -19.97 & -21.16 & -19.96 & -20.85 & -19.69 & -20.77 & -19.66 \\
\hline \multicolumn{9}{|c|}{ Mentally Unhealthy Days } \\
\hline 0 day & 1.11 & 1.77 & 1.12 & 1.80 & 1.30 & 2.00 & 1.34 & 1.99 \\
\hline $1-10$ days & -0.24 & 0.52 & -0.22 & 0.54 & -0.31 & 0.43 & -0.26 & 0.44 \\
\hline 11-20 days & -6.09 & -5.05 & -6.02 & -5.06 & -6.39 & -5.50 & -6.32 & -5.47 \\
\hline 21-29 days & -10.32 & -8.97 & -10.18 & -9.01 & -10.91 & -9.88 & -10.83 & -9.82 \\
\hline 30 days & -14.79 & -13.17 & -14.60 & -13.23 & -15.42 & -14.24 & -15.31 & -14.15 \\
\hline \multicolumn{9}{|c|}{ Activity Limitation Days } \\
\hline 0 day & 1.58 & 2.30 & 1.60 & 2.32 & 1.80 & 2.51 & 1.84 & 2.51 \\
\hline $1-10$ days & -2.00 & -1.20 & -1.97 & -1.19 & -1.95 & -1.19 & -1.90 & -1.18 \\
\hline $11-20$ days & -11.18 & -10.19 & -11.12 & -10.17 & -10.93 & -10.02 & -10.87 & -9.99 \\
\hline 21-29 days & -17.33 & -16.22 & -17.24 & -16.20 & -16.98 & -15.97 & -16.93 & -15.95 \\
\hline 30 days & -22.14 & -21.01 & -22.04 & -20.96 & -21.57 & -20.51 & -21.52 & -20.49 \\
\hline
\end{tabular}

Summary score with larger value means better quality of $\mathrm{HRQOL}$

Table 4 Summary of fit statistics for the one-factor HRQOL model over time

\begin{tabular}{lllllll}
\hline BRFSS Data Sources & $X^{2}$ & RMSEA & CFI & TLI & SRMR & CD \\
\hline $2001-2010$ (10 years) & 5441 & 0.041 & 0.987 & 0.924 & 0.012 & 0.880 \\
2001 & 248 & 0.035 & 0.989 & 0.933 & 0.010 & 0.868 \\
2002 & 126 & 0.036 & 0.986 & 0.918 & 0.011 & 0.873 \\
2003 & 319 & 0.035 & 0.99 & 0.937 & 0.010 & 0.880 \\
2004 & 448 & 0.039 & 0.988 & 0.928 & 0.011 & 0.881 \\
2005 & 499 & 0.038 & 0.989 & 0.934 & 0.011 & 0.877 \\
2006 & 519 & 0.039 & 0.989 & 0.933 & 0.011 & 0.881 \\
2007 & 720 & 0.042 & 0.988 & 0.926 & 0.012 & 0.883 \\
2008 & 665 & 0.041 & 0.988 & 0.929 & 0.011 & 0.879 \\
2009 & 793 & 0.044 & 0.987 & 0.920 & 0.012 & 0.879 \\
2010 & 1090 & 0.050 & 0.984 & 0.902 & 0.014 & 0.884 \\
$2011-2013$ (3 years) & 2670 & 0.044 & 0.987 & 0.924 & 0.012 & 0.884 \\
2011 & 991 & 0.045 & 0.986 & 0.917 & 0.012 & 0.883 \\
2012 & 978 & 0.046 & 0.986 & 0.915 & 0.012 & 0.883 \\
2013 & 715 & 0.039 & 0.990 & 0.938 & 0.011 & 0.885 \\
\hline All & &
\end{tabular}

All chi-square tests have 1 degree of freedom (df). RMSEA, Root mean squared error of approximation; $\mathrm{CFI}$, Comparative fit index; TLI, Tucker-Lewis index; SRMR, Standardized root mean squared residual; $C D$, Coefficient of determination would be simpler, more comprehensive, and more representative than using the four individual CDC HRQOL-4 items. Using this new measure, we found that overall HRQOL decreased in the US from 2001 to 2014. This trend may have started even earlier: an analysis of data from BRFSS and NHANES from 1993 to 2001 also found gradual decreases in health-related quality of life among adults, as indicated by several measures [11].

This study has several limitations. First, our measures of HRQOL were based solely on CDC's four core questions, which provide limited details about mental health symptoms. Second, the CDC HRQOL-4 questions are ordinal variables, which may have resulted in lower variance than would have existed had the variable been continuous. Thirdly, due to the large sample size, the chi-square test was not appropriate for our goodness of fit and model stability analyses. (The use of large samples can lead to significant p-values even if differences are small and meaningless. [32].) Instead, we used a list of other suitable statistics, such as RSMEA, CFI, and SMRM, to support our conclusions. Lastly, the study used self-reported data from BRFSS, which is subject to 
Table 5 Multi-group confirmatory factor analysis for measurement invariance across years, sex, and age subgroups

\begin{tabular}{|c|c|c|c|c|c|c|c|}
\hline Models & $x^{2}(d f)$ & RMSEA & $\mathrm{CFI}$ & TLI & SRMR & $C D$ & $\Delta \mathrm{CFI}$ \\
\hline \multicolumn{8}{|c|}{ BRFSS 2001-2010 (year subgroups) } \\
\hline Full configural invariance & $5430(10)$ & 0.041 & 0.987 & 0.925 & 0.011 & 0.88 & - \\
\hline Full metric invariance & $5648(37)$ & 0.022 & 0.987 & 0.979 & 0.012 & 0.88 & 0 \\
\hline Full scalar invariance & $10107(64)$ & 0.022 & 0.977 & 0.978 & 0.014 & 0.879 & 0.01 \\
\hline \multicolumn{8}{|c|}{ BRFSS 2011-2013 (year subgroups) } \\
\hline Full configural invariance & $2685(3)$ & 0.044 & 0.987 & 0.923 & 0.012 & 0.884 & - \\
\hline Full metric invariance & $2696(9)$ & 0.025 & 0.987 & 0.974 & 0.012 & 0.884 & 0 \\
\hline Full scalar invariance & $2794(15)$ & 0.02 & 0.987 & 0.984 & 0.012 & 0.884 & 0 \\
\hline \multicolumn{8}{|c|}{ BRFSS 2001-2010 (male vs. female) } \\
\hline Full configural invariance & $10443(2)$ & 0.057 & 0.997 & 0.981 & 0.01 & 0.879 & - \\
\hline Full metric invariance & $12243(5)$ & 0.039 & 0.996 & 0.991 & 0.012 & 0.879 & 0.001 \\
\hline Full scalar invariance & $23654(8)$ & 0.043 & 0.993 & 0.989 & 0.019 & 0.881 & 0.003 \\
\hline \multicolumn{8}{|c|}{ BRFSS 2011-2013 (male vs. female) } \\
\hline Full configural invariance & $5159(2)$ & 0.06 & 0.997 & 0.98 & 0.011 & 0.884 & - \\
\hline Full metric invariance & $5788(5)$ & 0.041 & 0.996 & 0.991 & 0.012 & 0.884 & 0.001 \\
\hline Full scalar invariance & $9425(8)$ & 0.041 & 0.994 & 0.991 & 0.017 & 0.885 & 0.002 \\
\hline \multicolumn{8}{|c|}{ BRFSS 2001-2010 (young vs. old ages) } \\
\hline Full configural invariance & $3587(2)$ & 0.034 & 0.999 & 0.994 & 0.007 & 0.877 & - \\
\hline Partial metric invariance & $13086(3)$ & 0.053 & 0.996 & 0.984 & 0.014 & 0.87 & 0.003 \\
\hline Full metric invariance & $62559(5)$ & 0.089 & 0.981 & 0.955 & 0.038 & 0.866 & 0.018 \\
\hline \multicolumn{8}{|c|}{ BRFSS 2011-2013 (young vs. old ages) } \\
\hline Full configural invariance & $2256(2)$ & 0.04 & 0.999 & 0.991 & 0.008 & 0.883 & - \\
\hline Partial metric invariance & $7096(3)$ & 0.058 & 0.996 & 0.982 & 0.013 & 0.879 & 0.003 \\
\hline Full metric invariance & $29614(5)$ & 0.092 & 0.981 & 0.955 & 0.035 & 0.874 & 0.018 \\
\hline
\end{tabular}

Full configural invariance - same factor structure across groups; full metric invariance-equivalent factor loadings across groups; full scalar invariance - equivalent intercepts across groups; partial metric invariance - equivalent factor loadings across groups except factor loadings for mentally unhealthy day item. Age subgroups: young (18-64) and old (65+)

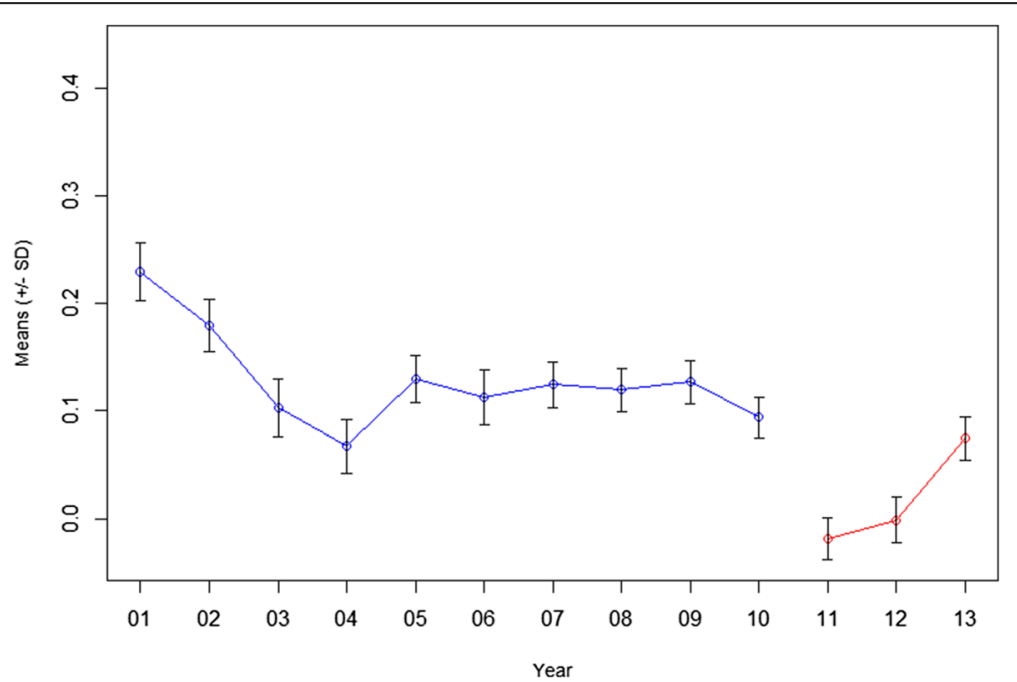

Fig. 3 Trend analysis of overall HRQOL, BRFSS 2001-2010 and 2011-2013. The weighted and age-adjusted HRQOL summary scores were predicated by the model for the 2001-2010 and 2011-2013 periods, respectively. The mean HRQOL summary score for each year is shown from 2001 to 2013. The 2000 US Census population was used for age standardization. 
recall and social desirability biases, as well as nonresponse bias due to the exclusion of persons not living in a private residence.

Our model has several advantages: (1) it can be broadly used by public health professionals, as the CDC HRQOL-4 questions are included in several national survey systems including BRFSS and NHANES; (2) it provides one-factor score values that could represent HRQOL at both the individual and population levels; and (3) it exhibits strong measurement invariance or stability over time, which makes it suitable for trend monitoring. Public health professionals may also apply similar factor analyses to other state- or community-level data sets for local health research, assessment, and evaluation. Finally, though our analysis indicates the value of a summary factor score for overall HRQOL, the collection and application of the CDC-HRQOL-4 items still remain to be important for studying HRQOL, especially when focusing on more specific aspects of HRQOL (e.g., physical health or mental health).

\section{Conclusion}

This study developed and tested a one-factor HRQOL model based on the CDC HRQOL-4 core questions. Using BRFSS data sets from 2001 to 2013, we evaluated the new model's goodness of fit, validity, stability, and measurement invariance over time. We also demonstrated the application of the predicated HRQOL factor score in trend analysis. These results suggest that it is feasible to apply the CDC HRQOL-4 core questions to study HRQOL through factor analysis with one underlying construct. The resulting summary score of HRQOL may be applied to health evaluation, subgroup targeting, trend monitoring, and risk factor identification.

\section{Additional file}

Additional file 1: Weighted Prevalences of Health-related Quality of Life Measures by Year, BRFSS. (DOCX $20 \mathrm{~kb}$ )

\section{Acknowledgments}

The authors thank Srila Sen and Magdala Labre for their editorial assistance. The authors also acknowledge suggestions from Diane Orenstein.

\section{Authors' contributions}

SY conceived the study, carried out the analysis, and drafted the manuscript. $R N, L B, P S$, and $L Y$ aided in conceiving the study, interpreting the results, and drafting the manuscript. All authors read and approved the final manuscript.

\section{Competing interests}

The authors declare that they have no competing interests.

\section{Disclaimer}

The findings and conclusions in this paper are those of the authors and do not necessarily represent the official position of the Centers for Disease Control and Prevention.

\section{Author details}

${ }^{1}$ SciMetrika, LLC, 100 Capitola Drive, Durham, NC 27701, USA. ${ }^{2}$ Centers for Disease Control and Prevention, 4770 Buford Highway NE, Atlanta, GA 30341, USA.

Received: 23 February 2016 Accepted: 21 June 2016

Published online: 11 July 2016

\section{References}

1. Palermo TM, Long AC, Lewandowski AS, Drotar D, Quittner AL, Walker LS. Evidence-based assessment of health-related quality of life and functional impairment in pediatric psychology. J Pediatr Psychol. 2008;33:983-96. discussion 997-988.

2. Revicki DA, Kleinman L, Cella D. A history of health-related quality of life outcomes in psychiatry. Dialogues Clin Neurosci. 2014;16:127-35.

3. Centers for Disease Control and Prevention. Measuring Healthy Days: Population assessment of health-related quality of life. Atlanta, Georgia: CDC. 2000. https://www.cdc.gov/hrqol/pdfs/mhd.pdf. Accessed Nov 2000.

4. Hennessy $\mathrm{CH}$, Moriarty DG, Zack MM, Scherr PA, Brackbill R. Measuring health-related quality of life for public health surveillance. Public Health Rep. 1994;109:665-72.

5. Moriarty DG, Zack MM, Kobau R. The Centers for Disease Control and Prevention's Healthy Days Measures - population tracking of perceived physical and mental health over time. Health Qual Life Outcomes. 2003;1:37.

6. Aaronson N, Alonso J, Burnam A, Lohr KN, Patrick DL, Perrin E, Stein RE. Assessing health status and quality-of-life instruments: attributes and review criteria. Qual Life Res. 2002;11:193-205.

7. Mielenz T, Jackson E, Currey S, DeVellis R, Callahan LF. Psychometric properties of the Centers for Disease Control and Prevention Health-Related Quality of Life (CDC HRQOL) items in adults with arthritis. Health Qual Life Outcomes. 2006; $4: 66$

8. Zullig KJ, Valois RF, Huebner ES, Drane JW. Evaluating the performance of the Centers for Disease Control and Prevention core Health-Related Quality of Life scale with adolescents. Public Health Rep. 2004;119:577-84.

9. Lackner JM, Gudleski GD, Zack MM, Katz LA, Powell C, Krasner S, Holmes E, Dorscheimer K. Measuring health-related quality of life in patients with irritable bowel syndrome: can less be more? Psychosom Med. 2006;68:312-20.

10. Wheaton AG, Ford ES, Thompson WW, Greenlund KJ, Presley-Cantrell LR, Croft JB. Pulmonary function, chronic respiratory symptoms, and health-related quality of life among adults in the United States-National Health and Nutrition Examination Survey 2007-2010. BMC Public Health. 2013:13:854.

11. Barile JP, Reeve BB, Smith AW, Zack MM, Mitchell SA, Kobau R, Cella DF, Luncheon C, Thompson WW. Monitoring population health for Healthy People 2020: evaluation of the NIH PROMIS(R) Global Health, CDC Healthy Days, and satisfaction with life instruments. Qual Life Res. 2013;22:1201-11.

12. Andresen EM, Fouts BS, Romeis JC, Brownson CA. Performance of healthrelated quality-of-life instruments in a spinal cord injured population. Arch Phys Med Rehabil. 1999;80:877-84.

13. Toet J, Raat H, van Ameijden EJ. Validation of the Dutch version of the CDC core healthy days measures in a community sample. Qual Life Res. 2006;15:179-84

14. Zahran HS, Kobau R, Moriarty DG, Zack MM, Holt J, Donehoo R, Centers for Disease C, Prevention. Health-related quality of life surveillance-United States, 1993-2002. MMWR Surveill Summ. 2005;54:1-35.

15. Zullig KJ. Creating and using the CDC HRQOL healthy days index with fixed option survey responses. Qual Life Res. 2010;19:413-24.

16. Nunnally JC. Psychometric Theory. 2nd ed. New York: McGraw-Hill; 1978.

17. Horner-Johnson W, Krahn G, Andresen E, Hall T, Rehabilitation R, Training Center Expert Panel on Health Status M. Developing summary scores of health-related quality of life for a population-based survey. Public Health Rep. 2009;124:103-10.

18. Raykov T. Reliability if deleted, not 'alpha if deleted': evaluation of scale reliability following component deletion. Br J Math Stat Psychol. 2007;60:201-16

19. Raykov T. Alpha if item deleted: a note on loss of criterion validity in scale development if maximizing coefficient alpha. Br J Math Stat Psychol. 2008; 61:275-85.

20. Remington PL, Smith MY, Williamson DF, Anda RF, Gentry EM, Hogelin GC. Design, characteristics, and usefulness of state-based behavioral risk factor surveillance: 1981-87. Public Health Rep. 1988;103:366-75. 
21. CDC. Methodologic changes in the Behavioral Risk Factor Surveillance System in 2011 and potential effects on prevalence estimates. MMWR Morb Mortal Wkly Rep. 2012;61:410-3.

22. Guttman L. Some necessary conditions for common factor analysis. Psychometrika. 1954;19:149-61.

23. Costello AB, Osborne JW. Best practices in exploratory factor analysis: Four recommendations for getting the most from your analysis. Pract Assess Res Eval. 2005;10:1-9.

24. Flora DB, Curran PJ. An empirical evaluation of alternative methods of estimation for confirmatory factor analysis with ordinal data. Psychol Methods. 2004;9:466-91.

25. Hu L-t, Bentler PM. Cutoff criteria for fit indexes in covariance structure analysis: Conventional criteria versus new alternatives. Struct Equ Model. 1999;6:1-55.

26. Milfont $T L$, Fischer R. Testing measurement invariance across groups: Applications in cross- cultural research. Int J Psychological Res. 2010;3:111-21.

27. Cheung GW, Rensvol RB. Evaluating goodness-of-fit indexes for testing measurement invariance. Struct Equ Model. 2002;9:233-55.

28. Vandenberg RJ, Lance CE. A Review and Synthesis of the Measurement Invariance Literature: Suggestions, Practices, and Recommendations for Organizational Research. Organ Res Methods. 2000;3:4-70.

29. Rubio DM, Gillepsie DF. Problems with error in structural equation models. Struct Equ Model. 1995;2:367-78.

30. Pons D, Atienza FL, Balaguer I, Garcia-Merita ML. Satisfaction with life scale: analysis of factorial invariance for adolescents and elderly persons. Percept Mot Skills. 2000;91:62-8.

31. Clench-Aas J, Nes RB, Dalgard OS, Aaro LE. Dimensionality and measurement invariance in the Satisfaction with Life Scale in Norway. Qual Life Res. 2011;20: 1307-17.

32. Bentler PM, Bonett DG. Significance tests and goodness of fit in the analysis of covariance structures. Psychol Bull. 1980;88:588-606.

\section{Submit your next manuscript to BioMed Central and we will help you at every step:}

- We accept pre-submission inquiries

- Our selector tool helps you to find the most relevant journal

- We provide round the clock customer support

- Convenient online submission

- Thorough peer review

- Inclusion in PubMed and all major indexing services

- Maximum visibility for your research

Submit your manuscript at www.biomedcentral.com/submit

C Biomed Central 